\title{
DISTRIBUIÇÃO ESPACIAL DE BROMÉLIAS NA RESTINGA DE JURUBATIBA, MACAÉ, RJ
}

\author{
André F. N. Freitas ${ }^{1.2}$ \\ Luciana Cogliatti-Carvalho ${ }^{1.2}$ \\ Monique Van Sluys ${ }^{1}$ \\ Carlos Frederico D. Rocha ${ }^{1}$
}

Recebido em 27/07/1999. Aceito em 24/02/2000

\begin{abstract}
RESUMO - (Distribuição espacial de bromélias na restinga de Jurubatiba, Macaé, RJ) - Nas restingas, devido aos microhábitats favoráveis, como as moitas, não ocorrerem de forma homogênea, mas em manchas, e devido à elevada taxa de reprodução vegetativa em bromélias, testa-se a hipótese de que o padrão de distribuição espacial das bromélias em uma restinga seria agregado. Utiliza-se como área para o teste de hipótese a restinga do Parque Nacional da Restinga de Jurubatiba. Analisa-se o padrão de distribuição espacial de cada espécie encontrada através do Índice de dispersão de Morisita (Id). Encontra-se 10 espécies de bromélias, com 10179 indivíduos em 0,98 ha amostrados. Na área estudada, nove espécies possuíram um padrão de distribuição agregado (Id $>1$ ). Sugerimos que o padrão de distribuição agregado de bromélias em restinga pode ser explicado pela taxa de reprodução vegetativa, distribuição de condições abióticas favoráveis e substratos disponíveis nas moitas, as quais ocorrem em manchas na restinga estudada, favorecendo a ocorrência de bromélias.
\end{abstract}

Palavras-chave - Distribuição agregada, reprodução vegetativa e manchas de moitas

ABSTRACT - (Spatial distribution of bromeliads at "restinga" of Jurubatiba, Macaé, RJ) - In "restingas", because favorable microhabitats have patchy distribution, and due to the high vegetative reproduction rate in bromeliads, we tested the hypothesis that the pattern of spatial distribution of bromeliads in a "restinga" habitat would be aggregated. We used the "restinga" of the Parque Nacional da Restinga de Jurubatiba as our hypothesis test area. We analyzed the spatial distribution pattern of each studied species using Morisita dispersion Index (Id). We found 10 bromeliad species, with 10179 individuals in the 0,98 ha sampled. In the study area, nine species had an aggregated distribution pattern (Id $>1$ ). We suggest that the aggregated distribution pattern of bromeliads may be explained by the rate of vegetative reproduction, distribution of the favorable abiotic conditions and availability substrates in clumps, which occur in patches in the study area, favoring the bromeliads occurrence.

Key-words - Aggregated distribution, vegetative reproduction and clumps patches

1 Universidade do Estado do Rio de Janeiro, Instituto de Biologia Roberto Alcântara Gomes, DBAV, Setor de Ecologia, Rua São Francisco Xavier, 524, CEP 20559-900, Maracanã, Rio de Janeiro, RJ, Brasil

2 Programa de Pós-Graduação em Ecologia da Universidade Federal do Rio de Janeiro, RJ, Brasil 


\section{Introdução}

O padrão de distribuição espacial de bromeliáceas em um determinado hábitat é determinado por fatores intrínsecos e extrínsecos à planta, tais como o mecanismo de reprodução utilizado pelo vegetal (Wilbur 1977; Henriques et al. 1984; Crawley \& May 1987), a presença de substrato apropriado (Fischer 1994) e as condições de luminosidade, temperatura e umidade favoráveis no microhábitat (Pittendrigh 1948; Johanson 1974; Sugden 1979; Ackerman 1986; Almeida 1997; Cogliatti-Carvalho 1999). Em hábitats desfavoráveis e com solos pobres em nutrientes, como as restingas (Henriques et al. 1986), a distribuição espacial das espécies vegetais pode ser influenciada pela capacidade particular de cada planta em resistir aos efeitos dos fatores ambientais limitantes, tais como salsugem, elevadas temperaturas do ar e do solo e alta incidência de luz solar, os quais irão determinar a comunidade vegetal local (Franco et al. 1984; Henriques et al. 1984; Araújo et al. 1998).

Como nas restingas os microhábitats favoráveis, tais como a borda ou o interior de moitas (onde o ambiente para o estabelecimento e o desenvolvimento de bromélias seria supostamente menos desfavorável), não ocorrem de forma homogênea, mas sim em manchas, e também devido à elevada taxa de reprodução assexuada em bromélias, seria de se esperar que o padrão de distribuição espacial destas plantas em restingas fosse predominantemente agregado.

Neste estudo, visou-se compreender o padrão de distribuição espacial de uma comunidade de Bromeliaceae em uma área de restinga no litoral norte do Estado do Rio de Janeiro, e testar a hipótese de que o padrão de distribuição espacial das espécies de Bromeliaceae na área estudada seria, de forma geral, agregado. Para tal, visou-se responder às seguintes questões: i) Quais as espécies de Bromeliaceae ocorrentes na área estudada do Parque Nacional da Restinga de Jurubatiba? ii) Qual o padrão de distribuição espacial de cada uma das espécies de bromélias na área?

\section{Material e métodos}

Área de estudo - O estudo foi realizado em um local próximo à Lagoa de Carapebus, no Parque Nacional da Restinga de Jurubatiba $\left(22^{\circ}\right.$ 17' $740^{\prime \prime} \mathrm{S}$ e $\left.41^{\circ} 41^{\prime} 111^{\prime \prime} \mathrm{W}\right)$, Município de Macaé, localizado a aproximadamente $200 \mathrm{~km}$ a nordeste da cidade do Rio de Janeiro, Estado do Rio de Janeiro, sudeste do Brasil. A região é dominada por uma planície arenosa quaternária, que se estende desde o mar até aproximadamente $2 \mathrm{~km}$ em direção ao interior do continente, onde inicia o cordão de Mata (Araújo et al. 1998). Esta restinga pode ser subdividida em tipos diferentes de vegetação: a) Halófila e psamófila reptante; b) Arbustiva fechada de pós-praia; c) Arbustiva aberta de Clusia, d) Arbustiva aberta de Ericaceae; e) Mata periodicamente inundada; f) Mata permanentemente inundada; g) Mata de cordão arenoso; h) Arbustiva aberta de Palmae; i) Herbácea brejosa e j) Aquática (ver Araújo et al. 1998). O clima da área é caracterizado como Tropical Sazonal, com uma estação de chuvas entre outubro e abril, e uma estação seca de maio a setembro. A pluviosidade média anual na área é de $1.200 \mathrm{~mm}$ e a temperatura média anual é de $22,6^{\circ} \mathrm{C}$ (Henriques et al. 1986; Zaluar 1997).

Metodologia - Estudou-se a comunidade de bromélias estabelecendo parcelas de $100 \mathrm{~m}^{2}(10$ x $10 \mathrm{~m})$ nas zonas halófila psamófila reptante (7 parcelas), fechada de pós-praia (14 parcelas), nas formações arbustivas abertas de Clusia (44 parcelas) e de ericácea ( 16 parcelas) e na mata periodicamente inundada (17 parcelas), durante o mês de março de 1999, analisando a distribuição espacial de cada espécie encontrada na área. As parcelas (total $=98$ ) foram delimitadas utilizando-se quatro estacas como vértices, as quais eram interligadas por um cordel. As parcelas foram estabelecidas em linha, a partir da região de praia (área halófila psamófila reptante) em 
direção ao interior, e com espaçamento de $10 \mathrm{~m}$ entre si. Toda a área interna de cada parcela foi cuidadosamente vistoriada, horizontal e verticalmente, sendo registradas as espécies de bromélias e o número de indivíduos de cada espécie encontrados, sendo estes de diferentes tamanhos, adultos e jovens de um tamanho mínimo cuja identificação fosse possível. Para estimar o padrão de distribuição espacial das diferentes espécies de bromélias na restinga como um todo e por zona, calculamos o Índice de dispersão de Morisita (Id) (Brower \& Zar 1984) para cada espécie separadamente:

$$
\mathrm{Id}=\mathrm{n} \frac{\Sigma \mathrm{X}^{2}-\mathrm{N}}{\mathrm{N}(\mathrm{N}-1)}
$$

onde " $\mathrm{n}$ " é o número de parcelas amostradas, "N" é o número total de indivíduos encontrados em todas as " $n$ " parcelas, e " $\Sigma \mathrm{X}^{2}$ " é a soma dos quadrados do número de indivíduos nas parcelas amostradas. A dispersão dos indivíduos é considerada aleatória se $\mathrm{Id}=1$, é agregada se Id $>1$ e é regular se Id $<1$. A significância do valor do Id foi testada através da estatística F, com nível de confiança de 0,05 (Poole 1974).

\section{Resultados e discussão}

$\mathrm{Na}$ área estudada da restinga de Jurubatiba, encontramos um total de 10 espécies de bromélias, com 10179 indivíduos em 0,98 ha amostrados (Tab. 1). Os principais estudos incluindo informações sobre bromélias de restingas da costa brasileira são Silva \& Somner 1984, na restinga de Barra de Maricá, e Araújo et al. 1998, na restinga de Jurubatiba, ambos registrando seis espécies em cada área estudada. Portanto, a riqueza de espécies encontrada pelo presente estudo é superior à riqueza de espécies encontrada naquelas áreas. As espécies de bromélias mais abundantes na restinga de Jurubatiba foram Aechmea nudicaulis $(\mathrm{N}=$ $2814)$, Neoregelia cruenta $(\mathrm{N}=2388)$ e Tillandsia stricta $(\mathrm{N}=1587)$ (Tab. 1). Todas as espécies tiveram o valor do Índice de dispersão de Morisita significativamente $>1$, tanto na restinga como um todo quanto nas diferentes zonas separadamente, não havendo diferenças na distribuição espacial das espécies nas cinco fisionomias estudadas. Os valores do Índice de dispersão de Morisita (Id) calculados para cada espécie encontrada na área estudada estão sumarizados na Tab. 1.

Os valores do Índice de dispersão de Morisita encontrados indicam que, na restinga de Jurubatiba, nove espécies de bromélias tiveram um padrão de distribuição agregado. Sugere-se que este padrão de distribuição agregado das bromélias na área estudada ocorre em fun-

Tabela 1. Abundância, valor do índice de dispersão de Morisita (Id), padrão de distribuição e valor da estatística F para Id das espécies de Bromeliaceae encontradas no Parque Nacional da Restinga de Jurubatiba, município de Macaé, RJ. Todos os resultados foram significativos ao nível de $5 \%$.

\begin{tabular}{lcrcc}
\hline Espécie de Bromeliaceae & Abundância & \multicolumn{1}{c}{ Id } & \multicolumn{1}{c}{ F } & Distribuição espacial \\
\hline Bromelia antiacantha & 911 & 6,24 & 50,15 & Agregada \\
Aechmea nudicaulis & 2814 & 2,99 & 58,71 & Agregada \\
Aechmea bromeliiffolia & 657 & 15,88 & 101,63 & Agregada \\
Aechmea lingulata & 1122 & 5,55 & 53,58 & Agregada \\
Neoregelia cruenta & 2388 & 2,05 & 26,83 & Agregada \\
Vriesea neoglutinosa & 512 & 14,26 & 70,85 & Agregada \\
Tillandsia stricta & 1587 & 3,64 & 44,16 & Agregada \\
Tillandsia gardneri & 138 & 20,59 & 28,66 & Agregada \\
Tillandsia recurvata & 50 & 32,64 & 16,98 & Agregada \\
Tillandsia usneoides & $-*$ & $-*$ & $-*$ & $*$ \\
Total & 10179 & - & - & - \\
\hline
\end{tabular}

Id = Índice de dispersão de Morisita. Id > 1 = Distribuição agregada.

* Não foi possível calcular estes parâmetros para a espécie Tillandsia usneoides devido à dificuldade de contar os indivíduos nos tufos desta espécie. 
Ção da interação de fatores intrínsecos e extrínsecos à planta. A constância no padrão de distribuição agregado das bromélias localmente pode ser explicada por basicamente três fatores: i) distribuição em manchas dos potenciais microhábitats favoráveis ao estabelecimento das bromélias, decorrente da formação de moitas, ii) existência, em contrapartida, de ambientes comparativamente mais desfavoráveis na região entre-moitas, e iii) ocorrência de reprodução vegetativa nas espécies de bromeliáceas.

$\mathrm{O}$ ambiente de restinga é influenciado por diferentes fatores limitantes, tais como salsugem, elevadas temperaturas, baixa umidade do ar, vento e elevada incidência de luz solar (Henriques et al. 1984; 1986). O estabelecimento pioneiro de algumas espécies vegetais em áreas de restinga pode propiciar a formação de moitas ou criar condições adequadas para que novas espécies se estabeleçam, criando potenciais microhábitats favoráveis que facilitam a entrada e a permanência de outras espécies que não sobreviveriam em condições abióticas extremas (Henriques et al. 1984). A mortalidade de sementes que caem no solo arenoso desprovido de vegetação das restingas pode ser alta devido às elevadas temperaturas, que podem atingir valores acima de $50^{\circ} \mathrm{C}$ (Franco et al., 1984; Fialho 1990). Deste modo, as moitas existentes na restinga oferecem microhábitats mais favoráveis à germinação de sementes, ao estabelecimento e ao crescimento das espécies vegetais do que a areia nua fora delas (Fialho \& Furtado 1993), já que oferecem maior disponibilidade de nutrientes, umidade e temperatura mais amena em virtude da menor exposição aos raios solares devido ao sombreamento proporcionado pelas folhas e galhos (Zaluar 1997). Uma vez estabelecidas, as moitas constituem um ambiente altamente heterogêneo, oferecendo uma ampla variação vertical e horizontal de substratos para as espécies epífitas e terrestres que dispõem, neste ambiente, de diversos microhábitats com diferentes condições microclimáticas e edáficas para se estabelece- rem e sobreviverem, dentro ou na periferia das moitas (Pereira \& Araújo 1995; Zaluar 1997). Assim, o padrão de distribuição agregado das bromélias na área pode ser explicado, em parte, pela presença das moitas, já que estas distribuem-se em manchas na área de restinga. As espécies epífitas encontradas no presente estudo (Vriesea neoglutinosa, Tillandsia stricta, T. recurvata e $T$. gardneri), de modo geral, tiveram os maiores valores de Id, indicando que são distribuídas com elevado grau de agregação. Embora não tenhamos contado o número de indivíduos da epífita T. usneoides, impossibilitando calcular o valor do Id, esta espécie ocorreu de forma agregada em tufos dentro de moitas, em apenas duas parcelas. As bromélias epífitas, em geral, possuem uma ampla distribuição vertical e horizontal no hábitat influenciadas pela luminosidade, umidade e temperatura ambiente, ocupando uma ampla gama de tipos de substratos (Pittendrigh 1948; Aragão 1967; Benzing 1980; Reitz 1985; Ackerman 1986; Almeida 1997; Cogliatti-Carvalho 1999). Aechmea bromeliifolia foi uma exceção que, apesar de não ter ocorrido associada a moitas, ocorreu exclusivamente na área de mata periodicamente inundada, embora também de forma agregada. A ocorrência desta espécie neste hábitat provavelmente é determinada pela luminosidade e temperatura mais amenas e pela maior umidade, e sua distribuição espacial agregada ocorre, provavelmente, devido ao mecanismo de reprodução vegetativa, mais utilizado pela espécie na área (obs. pess. dos autores).

Os efeitos dos fatores ambientais sobre a comunidade vegetal, somados à baixa quantidade de nutrientes no solo, são agravados nas regiões entre-moitas que são, geralmente, destituídas de vegetação (Pereira \& Araújo 1995). Regiões desprovidas de vegetação atingem temperaturas mais elevadas durante o dia, e com maiores amplitudes diárias, já que a ausência da cobertura vegetal facilita a mistura do ar quente com o ar frio da noite (Franco et al. 1984). Tal fato torna o ambiente neste local menos fa- 
vorável do que o interior das moitas, dificultando o estabelecimento de várias espécies vegetais (Henriques et al. 1986). No presente estudo, encontrou-se um maior número de espécies de bromélias vegetando no interior ou na borda de moitas, principalmente as epífitas, quando comparado à região entre moitas, que possuía basicamente bromélias capazes de suportar elevada incidência de luz solar e calor, tais como Neoregelia cruenta e Aechmea nudicaulis (para estas espécies ocorreram os menores valores do Índice de dispersão de Morisita). Lacerda \& Hay (1982) demonstraram que $N$. cruenta possui tanto hábitos terrestres quanto epífitos na restinga de Maricá, possivelmente em resposta à elevada intensidade de luz solar para a germinação ou o crescimento da bromélia. Contudo, o fato da presença destas duas espécies heliófilas nas regiões entre moitas ser possível pelas sementes germinarem dentro de moitas, com os indivíduos alcançando esta região através do brotamento direcional dos descendentes, necessita de estudos futuros.

A distribuição espacial das bromeliáceas pode também ser determinada pelo mecanismo de reprodução vegetativa utilizado (Wilbur 1977; Henriques et al. 1984; Crawley \& May 1987), característico de ocorrer em elevada taxa nesta família (ver Baracho 1997). Segundo Wikberg (1995), a distribuição espacial dos genets pode ser limitada pelo clima, pela distribuição de hábitats adequados e pela habilidade de dispersão do vegetal. Em ambientes hostis (como as restingas), ou que sofreram algum tipo de perturbação antrópica, como queimadas ou desmatamento, plantas que se reproduzem vegetativamente possuem melhores chances de sobreviver (Janzen 1980; Cook 1983; Freitas 1997), já que a reprodução assexuada garante aos indivíduos formarem descendentes escapando da esterilidade ou da falta de polinizadores. A reprodução vegetativa, ou em módulos, tende, então, a resultar em um padrão de distribuição agregado, por proporcionar um espalhamento da espécie sobre o substrato
(Henriques et al. 1984; Crawley 1990).

Conclui-se que o padrão de distribuição espacial das dez espécies de bromélias encontradas na área do Parque Nacional da Restinga de Jurubatiba é agregado. Este padrão de distribuição das bromélias parece estar associado à interação da estratégia reprodutiva assexuada, freqüentemente utilizada pelas espécies, e à ocorrência de moitas em manchas, que amenizam as condições limitantes de temperatura e luminosidade e constituem microhábitats favoráveis ao estabelecimento de Bromeliaceae.

\section{Agradecimentos}

Este estudo é parte dos resultados do Programa de Ecologia, Conservação e Manejo de Ecossistemas do Sudeste Brasileiro e do Projeto Vertebrados do Leste Brasileiro, Setor de Ecologia, Instituto de Biologia Roberto Alcântara Gomes, da Universidade do Estado do Rio de Janeiro. E. Leme e A. Costa identificaram as espécies de bromeliáceas. Durante o estudo, A. F. N. Freitas (CAPES) e L. Cogliatti-Carvalho (CNPq) receberam bolsas de Mestrado. C. F. D. Rocha (Processo No 300 819/94-3) e M. Van Sluys (Processo No 301117/95-0) receberam bolsas de Produtividade em Pesquisa do Conselho Nacional do Desenvolvimento Científico e Tecnológico - CNPq. Este estudo foi subvencionado com auxílio à Pesquisa do $\mathrm{CNPq}$ para $\mathrm{C}$. F. D. Rocha.

\section{Referências Bibliográficas}

Ackerman, J. D. 1986. Coping with the epiphytic existence: pollination strategies. Selbyana 9: 52-60.

Almeida, D. R. 1997. Composição, riqueza e diversidade das comunidades de bromeliáceas em diferentes ambientes da área de Mata Atlântica da Vila Dois Rios, Ilha Grande, RJ. Monografia de Bacharelado. Universidade do Estado do Rio de Janeiro, RJ.

Aragão, M. B. 1967. Condições de hábitat e distribuição geográfica de algumas Bromeliaceae. Sellowia 19: 83-95. 
Araújo, D. S. D.; Scarano, F. R.; Sá, C. F. C.; Kurtz, B. C.; Zaluar, H. L. T.; Montezuma, R. C. M. \& Oliveira, R. C. 1998. Comunidades Vegetais do Parque Nacional da Restinga de Jurubatiba. Pp. 39-62. In: F. A. Esteves (Ed.) Ecologia das Lagoas Costeiras do Parque Nacional da Restinga de Jurubatiba e do Município de Macaé (RJ). Universidade Federal do Rio de Janeiro, Rio de Janeiro.

Baracho, G. S. 1997. Propagação vegetativa em Bromeliaceae. Bromélia 4(2): 23-28.

Benzing, D. H. 1980. The Biology of Bromeliads. Mad River Press, California.

Brower, J. E. \& Zar, J. H. 1984. Field \& Laboratory methods for general ecology. Wm. C. Brown Publishers, Dubuque, Iowa.

Cogliatti-Carvalho, L. 1999. Distribuição espacial e substrato preferencial de Neoregelia johannis (Bromeliaceae) em uma área de Floresta Atlântica secundária da Ilha Grande, RJ. Monografia de Bacharelado. Universidade do Estado do Rio de Janeiro, RJ.

Cook, R. E. 1983. Clonal plant populations. American Scientist 71: 244-253.

Crawley, M. J. \& May, R. M. 1987. Population dynamics and plant community structure: competition between annuals and perennials. Journal of theoretical Biology 125: 475-489.

Crawley, M. J. 1990. The population dynamics of plants. Philosophy Transactions of the Royal Society of London B 330: 125-140.

Fialho, R. F. 1990. Seed dispersal by a lizard and a treefrog - effect of dispersal site on seed survivorship. Biotropica 22(4): 423-424.

Fialho, R. F. \& Furtado, A. L. S. 1993. Germination of Erythroxylum ovalifolium (Erythroxylaceae) seeds within the terrestrial bromeliad Neoregelia cruenta. Biotropica 25(3): 359-362.

Fischer, E. 1994. Polinização, fenologia e distribuição espacial de bromeliaceae numa comunidade de Mata Atlântica, litoral sul de São Paulo. Dissertação de Mestrado. Universidade Estadual de Campinas, SP.

Franco, A. C.; Valeriano, D. M.; Santos, F. M.; Hay, J. D.; Henriques, R. P. B. \& Medeiros, R. A. 1984. Os microclimas das zonas de vegetação da praia da restinga de Barra de Maricá, Rio de Janeiro. Pp. 413425. In: L. D. Lacerda, D. S. D. Araújo, R. Cerqueira \& B. Turcq (Eds.), Restingas: Origem, estrutura e processos. CEUFF, Niterói.

Freitas, C. A. 1997. Crescimento clonal em duas espécies de Nidularium (Bromeliaceae) sobre solo inundado em Mata Atlântica. Dissertação de Mestrado. Universidade Federal do Rio de Janeiro, Rio de Janeiro.
Henriques, R. P. B.; Meirelles, M. L. \& Hay, J. D. 1984. Ordenação e distribuição de espécies das comunidades vegetais na praia da restinga de Barra de Maricá, Rio de Janeiro. Revista Brasileira de Botânica 7(1): 27-36.

Henriques, R. P. B.; Araújo, D. S. D. \& Hay, J. D. 1986. Descrição e classificação dos tipos de vegetação da restinga de Carapebus, Rio de Janeiro. Revista Brasileira de Botânica 9(2): 173-189.

Janzen, D. H. 1980. Ecologia Vegetal nos Trópicos. v. 7, Coleção temas de Biologia, 79 pp. EDUSP, São Paulo,

Johanson, D. 1974. Ecology of vascular epiphytes in west African rainforest. Acta Phytogeographical Succ 59:1-136.

Lacerda, L. D. \& Hay, J. D. 1982. Hábitat of Neoregelia cruenta (Bromeliaceae) in coastal sand dunes of Marica, Brazil. Revista de Biologia Tropical 171173.

Pereira, O. J. \& Araújo, D. S. D. 1995. Estrutura da vegetação de entre moitas da formação aberta de Ericaceae no Parque Estadual de Setiba, ES. Oecologia Brasiliensis 1: 245-257.

Pittendrigh, C. S. 1948. The bromeliad-anophelesmalaria complex in Trinidad I - the bromeliad flora. Evolution 2: 58-89.

Poole, R. W. 1974. An Introduction to Quantitative Ecology. McGraw-Hill, Inc. Nova Iorque, EUA.

Reitz, R. 1985. Na trama da malária. Ciência Hoje 4: 50-57.

Silva, J. G. \& Somner, G. V. 1984. A vegetação da restinga na Barra de Maricá, RJ. Pp. 217-232. In: L. D. Lacerda, D. S. D. Araújo, R. Cerqueira \& B. Turcq (Eds.), Restingas: Origem, estrutura e processos. CEUFF, Niterói.

Sugden, A. M. 1979. Aspects of the ecology of vascular epiphytes in Columbian cloud forest. I. The distribution of the epiphytic flora. Biotropica 11:173-188.

Wikberg, S. 1995. Fitness in clonal plants. Oikos 72(2): 293-297.

Wilbur, H. M. 1977. Propagule size, number, and dispersion pattern in Ambystoma and Asclepias. American Naturalist 111: 43-68.

Zaluar, H. L. T. 1997. Espécies focais e a formação de moitas na restinga aberta de Clusia. Dissertação de Mestrado. Universidade Federal do Rio de Janeiro, Rio de Janeiro. 